\title{
Autour des Cent Nouvelles nouvelles. Sources et rayonnements, contextes et interprétations
}

\section{Maria Colombo Timelli}

\section{(2) OpenEdition}

1 Journals

Édition électronique

URL : http://journals.openedition.org/studifrancesi/10525

DOI : 10.4000/studifrancesi. 10525

ISSN : 2421-5856

Éditeur

Rosenberg \& Sellier

\section{Édition imprimée}

Date de publication : 1 décembre 2017

Pagination : 531-532

ISSN : 0039-2944

\section{Référence électronique}

Maria Colombo Timelli, « Autour des Cent Nouvelles nouvelles. Sources et rayonnements, contextes et interprétations », Studi Francesi [En ligne], 183 (LXI | III) | 2017, mis en ligne le 01 février 2018, consulté le 01 février 2021. URL : http://journals.openedition.org/studifrancesi/10525; DOI : https://doi.org/ 10.4000/studifrancesi. 10525

Ce document a été généré automatiquement le 1 février 2021.

\section{(c) (†) $\odot$}

Studi Francesi è distribuita con Licenza Creative Commons Attribuzione - Non commerciale - Non opere derivate 4.0 Internazionale. 


\title{
Autour des Cent Nouvelles nouvelles. Sources et rayonnements, contextes et interprétations
}

\author{
Maria Colombo Timelli
}

\section{RÉFÉRENCE}

Autour des Cent Nouvelles nouvelles. Sources et rayonnements, contextes et interprétations. Actes du colloque international organisé les 20 et 21 octobre 2011 à l'Université du Littoral - Côte d'Opale (Dunkerque). Textes édités par Jean DEVAux et Alexandra VELISSARIOU, Paris, Honoré Champion, 2016, «Bibliothèque du Xve siècle» 81, 318 pp.

1 La rencontre dunkerquoise de 2011, dont ce recueil représente les Actes, se proposait de faire le point sur deux questions fondamentales: les sources ayant inspiré l'auteur du recueil «bourguignon», et l'influence exercée par les CNN dans les siècles successifs, jusqu'à nos jours. Signalons que les Actes du colloque qui s'est déroulé à Glasgow en septembre 2011 également, centré sur le manuscrit Hunter 252, sont en revanche toujours en préparation.

2 C'est de main de maître que le regretté Jean DUFOURNET ouvre ce volume, en analysant les traits distinctifs des CNN: l'ambiguïté, poursuivie avec toutes sortes de ressources linguistiques, la tromperie, basée sur la parole, la centralité du corps et de l'acte sexuel en particulier, le rire enfin, qui permet de tout désacraliser. Comme le souligne Dufournet en conclusion, devant un monde dont le sens se dérobe sans cesse, «cette prise de conscience s'accomplit non dans le tragique, mais dans une allégresse opulente» (p. 29): c'est cette allégresse qui continue d'assurer aux CNN toute leur fraîcheur (Les CNN, emblème de la génération de Louis XI, pp. 15-29). En renonçant à traiter les CNN uniquement comme un texte littéraire, Jelle кOopmans propose de les situer dans un cadre ludique, les nouvelles reflétant un fait social, pratiqué en milieu bourguignon: à ses yeux, c'est dans un espace social qu'il faudra situer le recueil afin de 
lui restituer sa réalité historique (Espaces ludiques: le jeu de la sociabilité bourguignonne, pp.31-40). C'est le discours moralisateur émergeant de certaines nouvelles qui intéresse en revanche Jean DEVAUX; si dans certains contes la moralité est explicitée par le narrateur, dans d'autres elle n'est que suggérée par le récit: que ce soit sur le mode sérieux ou sur le parodique, l'exemplarité du recueil correspond bien à la "propension au didactisme» (p. 41) qui marque la production littéraire du Moyen Âge tardif («Qui est notable et veritable exemple»: les CNN et le didactisme bourguignon, pp. 41-52).

D'autres contributions se focalisent sur l'un ou l'autre conte. Profond connaisseur et du genre et de ce recueil, Roger DuBuis s'interroge sur l'avant-dernière nouvelle (la dernière dans le manuscrit de Glasgow), celle qui pose à ses yeux «le plus d'énigmes» (p.56): cela tient à sa longueur exceptionnelle d'une part, au traitement du sujet de l'autre, qui ne fait pas de place au «comique facile» (p. 66). Selon Dubuis, on trouve là une sorte de modèle du genre même, ainsi qu' «une véritable défence et illustration de la nouvelle» (p. 66) (L'énigme de la nouvelle du Vœeu du clerc, pp. 53-66). Auteur d'une grosse thèse sur la nouvelle médiévale (2006), Nelly LABÈRE applique aux trois dernières nouvelles la proposition méthodologique avancée par G. Deleuze et F. Guattari dans le chapitre 8 des Mille plateaux (1980). Elle met ainsi l'accent sur la discontinuité du recueil, sur la multiplication des voix et sur l'organisation circulaire de l'ensemble («Trois dernières nouvelles ou 'qu'est-ce qui s'est passé?'». Les Milles plateaux des CNN, pp. 67-82). Le couple adultère de la première nouvelle - une gouge anonyme et un bourgois plus subtil que ung regnard - renvoie nécessairement au couple Renard-Hersent dans la branche II du Roman de Renard de Pierre de Saint-Cloud. Brînduşa GRIGORIU propose donc une comparaison entre les deux textes sur la base de l'opposition devantderrière, fondamentale dans la conception et dans la perception de la femme médiévale (La face et le derrière: 'renardies' de la première nouvelle, pp. 135-147). La différenciation rigide des sexes dans les $C N N$ invite à s'interroger sur la 'masculinité'-objet relativement récent au sein des gender studies - au $\mathrm{XV}^{\mathrm{e}}$ siècle, notamment en milieu bourguignon. Selon David LAGUARDIA, la nouvelle 37 représente une réalisation frappante des traditions didactique et comique exploitées dans le recueil: les relations entre hommes et femmes s'y articulent tant dans l'espace physique que dans l'imaginaire (La représentation de la masculinité dans les CNN, pp. 149-163). Tovi BIBRING étudie le fonctionnement de la double trame narrative qui fonde la nouvelle 64, dont le sujet est proche de celui du Prêtre crucifié: de fait, la nouvelle se différencie du fabliau par l'absence d'une femme particulière et par le rapport entre la parole et la castration finale du curé (Farce et châtiment: lecture de la nouvelle 64 des CNN, pp. 165-176).

Des questions plus générales, thématiques, stylistiques ou encore linguistiques, sont abordés dans d'autres articles. À partir du constat de l'omniprésence du corps dans les CNN, Luca PIERDOMINICI analyse les jeux d'«abstraction» parsemés dans le texte: jeux de mots, antiphrases, accumulations, euphémismes, métaphores, qui tous sollicitent la participation du lecteur qui voudra en tirer le sens (La désincarnation du corps dans les CNN, pp. 83-102). Procédés des mieux étudiés dans les $C N N$, l'ironie retient encore l'attention de Evelio miÑANO MARTíNEZ, qui en rappelle quelques procédés (métaphore, métonymie, voire mensonge) et souligne l'importance de la participation active du lecteur, qui se doit de dépasser le niveau littéral du texte afin de parvenir à interpréter correctement les intentions du conteur et finalement de jouir du plaisir du rire (L'ironie dans les CNN: la clef d'un univers de fiction, pp.103-118). S'il est banal de relever l'importance de l'argent dans les CNN, Cristina AZUELA met plutôt l'accent sur l'attitude 
fortement ambiguë de l'auteur à l'égard des marchands et des bourgeois qui peuplent son recueil; celle-ci s'exprime notamment par un jeu constant entre lexique et imaginaire monétaire et sexuel (Paroles adultères, marchands et argent dans les CNN, pp. 119-134). Loin de refléter la provenance géographique des différents conteurs dont l'identité et l'origine sont avérées, la langue des $C N N$ se révèle sensiblement homogène et marquée par de nombreux picardismes. Ceci serait la preuve, selon Geoffrey ROGER, d'une «identité linguistique manifeste» à la cour de Bourgogne (p.192). Cette affirmation paraît néanmoins risquée en l'absence du manuscrit offert à Philippe le Bon, dans la mesure où les traits dialectaux décelés pourraient être le fait du copiste du manuscrit de Glasgow (La mise en scène des CNN: point de vue dialectologique sur le manuscrit Hunter 252, pp. 177-192).

Une partie du volume est enfin consacrée à la réception des $C N N$ à partir du siècle de la Renaissance. L'étude des titres et des péritextes des CNN dans les éditions imprimées à partir de la princeps de Vérard (1486) jusqu'à l'édition de Franklin P. Sweetser en 1996, menée par Alexandra VELISSARIOU, montre bien comment le recueil demeure attaché à Louis XI, et ce même après la découverte du manuscrit Hunter en 1854, qui associait indubitablement la composition du recueil à la cour de Philippe le Bon (Le paratexte des CNN, du Moyen Âge au XXe siècle: titres, préfaces et introductions, pp. 193-214). Hisara KONDO souligne le fait que Philippe de Vigneulles s'inspira certainement des CNN dans la composition de son recueil (rédigé entre 1505 et 1515); celui-ci est néanmoins ancré dans la réalité messine et surtout dans la manière d'écrire de Philippe, ce qui ressort en particulier de la comparaison entre les Nouvelles et certains passages des Mémoires et de la Chronique du même auteur (Les CNN bourguignonnes et Philippe de Vigneulles, pp. 215-225). Madeleine JEAY s'interroge ensuite sur l'usage que fit Bonaventure des Périers du fonds narratif des $C N N$. Si les rapports directs entre telle et telle nouvelle sont très limités en quantité (quatre nouvelles de Des Périers seraient à rattacher à six des $(N N)$, la référence implicite au recueil bourguignon, ainsi qu'au Décaméron de Boccace, demeure certaine (Lecture des CNN par Bonaventure des Périers, pp. 227-239). Les CNN ont également inspiré La Fontaine: les nouvelles 21, 32 et 37 en particulier ont fourni le sujet pour trois contes, examinés ici par Mathieu BERMANN. Réécrits en vers dans le contexte galant des années 1660-1670, les récits du Xve siècle sont pliés au goût $\mathrm{du}$ moment et se chargent-par des omissions autant que par des ajouts-d'une critique virulente, adressée aux médecins, au clergé, voire aux «Tartuffes» contemporains (Les CNN relues par La Fontaine: un intertexte licencieux, pp. 241-252).

6 Le volume se clôt par quatre «contes» inspirés aux CNN (nouvelles 69, 25, 11, 19), le premier en vers, les trois autres en prose, par Claude MASTRE: même si ces récits, renouvelés pour le lecteur-auditeur moderne, sont basés sur la traduction fournie en 1991 par Roger Dubuis, ils ne manquent pas de confirmer la fascination que l'ancien recueil continue d'exercer de nos jours (Il nous raconte des histoires!, pp. 253-299).

7 Danielle QUÉRUEL tire enfin le bilan des deux journées dunkerquoises, en rappelant surtout que, d'une part, l'intérêt pour les CNN elles-mêmes est relativement récent, d'autre part que les articles réunis ici ouvrent certainement des pistes de recherche renouvelées (Conclusion, pp. 301-306).

Un Index des noms et des titres est publié aux pp. 307-316. On regrette un peu l'absence d'une bibliographie, qui aurait pu mettre à jour et compléter celles - imposantes - de Nelly Labère (2006) et d'Alexandra Velissariou (2012). 